\title{
Damage effects and mechanism of GaAs solar cells induced by high-power microwaves
}

\author{
Hanxiang Wang ${ }^{1}$, Changchun Chai ${ }^{1}$, Yuqian Liu ${ }^{1}$, a) , Han $\mathrm{Wu}^{1}$, Wei Zhang ${ }^{1}$, Fuxing $\mathrm{Li}^{1}$, and Yintang Yang ${ }^{1}$
}

Abstract The two-dimensional thermoelectric models of single-junction and triple-junction GaAs solar cells are established respectively utilizing Sentaurus-TCAD, to investigate the damage effects caused by HPMs. Simulation results demonstrate that there are two burnout mechanisms of GaAs solar cells: damage caused by Joule heat accumulation under high electric field, and failure due to temperature surges induced by avalanche breakdown. In addition, fitted empirical formulas also show that burnout caused by Joule heat accumulation at the inflection point of the cathode front surface field occurs when the frequency of injection is above $3 \mathrm{GHz}$, and damage energy decreases as the frequency increases. In contrast, the avalanche multiplication effect in the reverse-biased space charge region near the back-surface field can be triggered when the frequency is below $3 \mathrm{GHz}$, and damage energy rises as the frequency rises. Besides, due to the enhancement of heat dissipation and the drop in avalanche ionization rate, the multi-junction GaAs solar cell becomes more difficult to burn out than the single-junction solar cell under the same HPM interference. Moreover, an equivalent model (based on the carrier mobility distribution when the injected HPMs signal does not reach the burnout threshold) is rebuilt to study the soft damage effect on the performance of GaAs solar cells, as caused by the injection of HPMs.

Keywords: GaAs solar cell, multi-junction, HPMs, frequency of injection, soft damage

Classification: Electron devices, circuits and modules (silicon, compound semiconductor, organic and novel materials)

\section{Introduction}

Due to the seriously wasted fossil energy (coal, oil \& gas) and safety problems of nuclear power, the concept of Space Solar Power Station (SSPS), known as "the Manhattan project of energy", has attracted unprecedented attention, which converts solar energy into electrical energy for the ground without the limitations of weather and darkness [1, 2, 3, 4, 5]. SSPS consists of three main parts: space solar power collection and photoelectric conversion, high-power microwave transmitting antenna, receiving and rectification $[6,7,8]$. Baoyan Duan's team of Xidian University verified the feasibility of the indoor mock-up of OMEGA concept and has been building the world's first full-link, full-system outdoor wireless high-power continuous microwave energy transmitting prototype $[9,10,11]$. NASA and ESA also plan to construct large-scale solar cell tile arrays and high-power microwave transmitting antennas at the equator in the fu-

${ }^{1}$ Wide Bandgap Semiconductor Technology Disciplines State Key Laboratory, Xidian University, Xi' an 710071, P.R. China

a) yuqianliuxd@163.com

DOI: $10.1587 /$ elex.18.20210020

Received January 14, 2021

Accepted February 1, 2021

Publicized February 9, 2021

Copyedited March 10, 2021 ture $[12,13,14]$. Above all, it is necessary to ensure the solar cells can work stably in high-power microwave environments.

Currently, theorical or experimental researches on the HPM damage effects of spacecraft mainly focus on the internal semiconductor devices and integrated circuits, while rarely involves solar cells, because HPMs is easily coupled into electronic systems through front-door paths (such as antennas, etc.) but difficult to be reversely injected into cells through back-door paths (such as transmission cables, etc.) $[15,16]$. However, as SSPS (a most important fourthgeneration new energy system) becomes popular and widely promoted in the future, more large-scale solar cell tile arrays and high-power microwave transmitting antennas (commercial output power can reach up to GW level) will be built together in space, which greatly increases the risk of solar cells being reversely injected by HPMs [17, 18, 19, 20, 21].

In this paper, the two-dimensional thermoelectric models of single-junction and triple-junction GaAs solar cells (a mainstream cell of space batteries [22]) respectively by using the Sentaurus-TCAD. The damage effects and mechanism are studied with the injection of HPMs in the cathodal of GaAs solar cells, and two burnout mechanisms are proposed: damage caused by Joule heat accumulation under high electric field, and failure due to temperature surges induced by avalanche breakdown, which can refer to the damage effects and mechanism of BJT, HEMT and CMOS induced by the injection of HPMs [23, 24]. In addition, the simulation results between two models are compared, which demonstrates that the difficulty of burning increases as the number of junctions increases. Moreover, mobility degradation effects on the performance of GaAs solar cells are also revealed. Above researches can help to explore the measures to protect GaAs solar cells from the attack of HPMs, further improving the reliability of SSPS.

\section{Structure and numerical model}

The two-dimensional structure models of GaAs solar cells are depicted respectively in Fig. 1(a) and Fig. 1(b). Besides, the details of thickness and doping levels from Fig. 1 (used in simulations) are specified in Table I.

The I-V and P-V curves of the optical simulation results are presented in Fig. 2. Note that these structure models are studied under the AM0 solar spectrum, with $P=1 \mathrm{~W} / \mathrm{cm}^{2}$, and at temperature $T=300 \mathrm{~K}$.

Due to the high magnetic field and strong current caused 
(a)

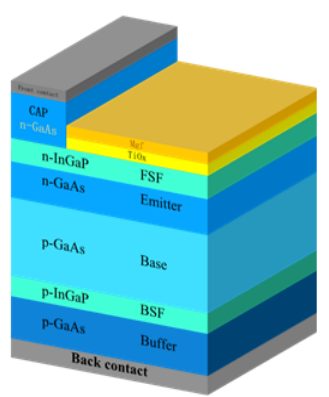

(b)

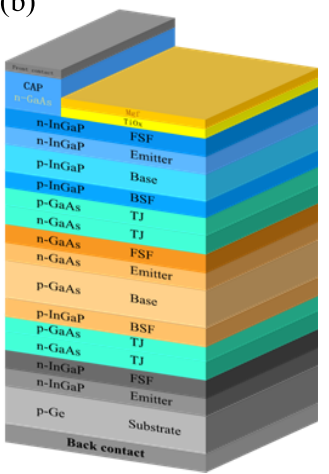

Fig. 1 The three-dimensional view of GaAs solar cells. (a) A singlejunction GaAs solar cell. (b) A triple-junction $\mathrm{InGaP} / \mathrm{GaAs} / \mathrm{Ge}$ solar cell.

Table I Details of the triple-junction GaAs solar cell in simulation.

\begin{tabular}{|c|c|c|c|c|}
\hline \multirow{2}{*}{ Cell } & Region & Material & Thickness $[\mu m]$ & Doping $\left[\mathrm{cm}^{-3}\right]$ \\
\hline & Cap & GaAs & 1 & $5.0 e^{18}$ \\
\hline \multirow{4}{*}{$\begin{array}{l}\text { InGaP Top } \\
\quad \text { cell }\end{array}$} & $\mathrm{fsf}$ & InGaP & 0.04 & $7.0 e^{18}$ \\
\hline & emitter & $\mathrm{InGaP}$ & 0.05 & $1.8 e^{18}$ \\
\hline & base & InGaP & 0.6 & $-1.2 e^{17}$ \\
\hline & bsf & InGaP & 0.03 & $-2.0 e^{18}$ \\
\hline \multirow{2}{*}{$\begin{array}{l}\text { Tunneling } \\
\text { diode }\end{array}$} & $\operatorname{tdp}$ & GaAs & 0.015 & $-1.0 e^{19}$ \\
\hline & $\operatorname{tdn}$ & GaAs & 0.015 & $4.0 e^{20}$ \\
\hline \multirow{4}{*}{$\begin{array}{c}\text { GaAs } \\
\text { Medium cell }\end{array}$} & fsf & $\mathrm{InGaP}$ & 0.03 & $7.0 e^{18}$ \\
\hline & emitter & GaAs & 0.1 & $2.0 e^{18}$ \\
\hline & base & GaAs & 1.5 & $-1.0 e^{17}$ \\
\hline & bsf & $\mathrm{InGaP}$ & 0.05 & $-2.0 \mathrm{e}^{18}$ \\
\hline \multirow{2}{*}{$\begin{array}{l}\text { Tunneling } \\
\text { diode }\end{array}$} & tdp & GaAs & 0.015 & $-1.0 e^{19}$ \\
\hline & $\operatorname{tdn}$ & GaAs & 0.015 & $4.0 e^{20}$ \\
\hline \multirow{3}{*}{$\begin{array}{l}\text { Germanium } \\
\text { bottom cell }\end{array}$} & $\overline{f s f}$ & $\mathrm{InGaP}$ & 0.03 & $7.0 e^{18}$ \\
\hline & emitter & $\mathrm{InGaP}$ & 0.1 & $1.8 e^{18}$ \\
\hline & substrate & Germanium & 0.5 & $-1.0 e^{17}$ \\
\hline
\end{tabular}

by the injection of HPMs, a huge heat is generated. Therefore, considering the effect of temperature gradients on carrier transport, the current density equation can be modified as follows [25]:

$$
\begin{aligned}
& J_{\mathrm{n}}=-n q \mu_{\mathrm{n}}\left(\nabla \phi_{\mathrm{n}}+P_{\mathrm{n}} \nabla T\right) \\
& J_{\mathrm{p}}=-p q \mu_{\mathrm{p}}\left(\nabla \phi_{\mathrm{p}}+P_{\mathrm{p}} \nabla T\right)
\end{aligned}
$$

where $P_{n}\left(P_{p}\right)$ is the electron (hole) absolute thermoelectric power. Additionally, an avalanche model, known as van Overstraeten-de Man model [26], describes the generation process of the electron-hole pair, so avalanche generation rate $G$ can be expressed as follows:

$$
G=\alpha_{n} n v_{n}+\alpha_{p} n v_{p}
$$

where $v_{n}\left(v_{p}\right)$ is the electron (hole) drift velocity, $\alpha_{n}\left(\alpha_{p}\right)$ donates the electron (hole) ionization rate.

Under high electric field, the carrier drift rate is inclined towards saturation velocity $\left(V_{\text {sat }}\right)$. The expression of the carrier mobility model considering high electric field saturation is shown as follow [27]:

$$
\mu(E)=\frac{\mu_{\text {low }}}{\left[1+\left(\mu_{\text {low }} E / v_{\text {sat }}\right)^{\beta}\right]^{1 / \beta}}
$$

where $\mu_{\text {low }}$ refers to the low field coupling mobility.

The inductive voltage coupling from the cathode wire (under HPM interference) is equivalent to a damped sinusoidal
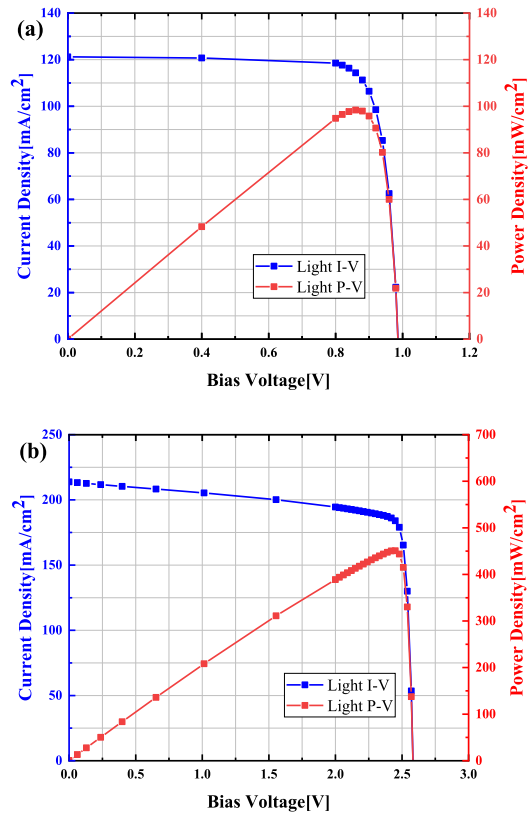

Fig. 2 (a) Light I-V and P-V characteristics of the single-junction GaAs solar cell. (b) Light I-V and P-V characteristics of the triple-junction InGaP/GaAs/Ge solar cell.

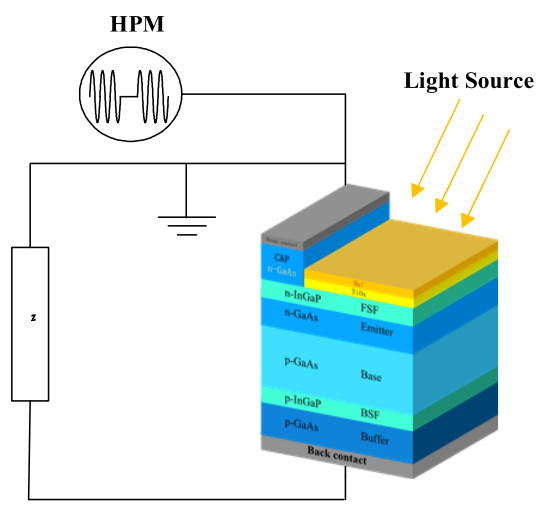

Fig. 3 Schematic diagram of the GaAs solar cell with the cathode port HPMs injection.

signal. The mathematical formula in this study is shown as follow:

$$
U=U_{0} \sin (2 \pi f t+\varphi)
$$

where $U_{0}$ represents the amplitude, $f$ is the frequency, and $\varphi$ is the initial phase. The device burns out when the peak lattice temperature reaches the melting point of GaAs at $1511 \mathrm{~K}$.

\section{Mechanism and discussion}

Considering the high conversion efficiency of $2.4 \mathrm{GHz}$ $(5.8 \mathrm{GHz})$ in wireless power transmission and the high damage threshold of multi-layer devices (such as super-junction power device) [28, 29, 30, 31], for the device shown in Fig. 1(a), a HPMs signal with amplitude of $120 \mathrm{~V}$ and frequency of $2 \mathrm{GHz}$ is reversely injected from the cathode port (Fig. 3). Two heat sources emerge near the front (back) surface field heterojunction alternately.

During the positive half cycle (when the amplitude of the injected wave is greater than zero), the front surface 


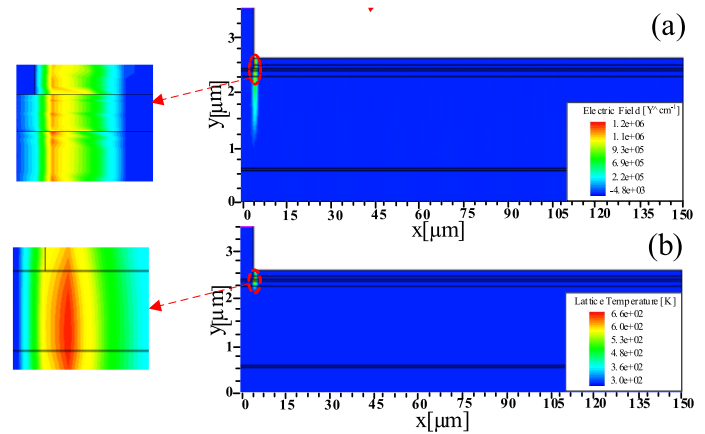

Fig. 4 The single-junction GaAs solar cell at $0.19 \mathrm{~ns}$ under the injection of HPMs signal with amplitude of $120 \mathrm{~V}$ and frequency of $2 \mathrm{GHz}$. (a) The electric field distribution inside the device. (b) The temperature distribution inside the device.
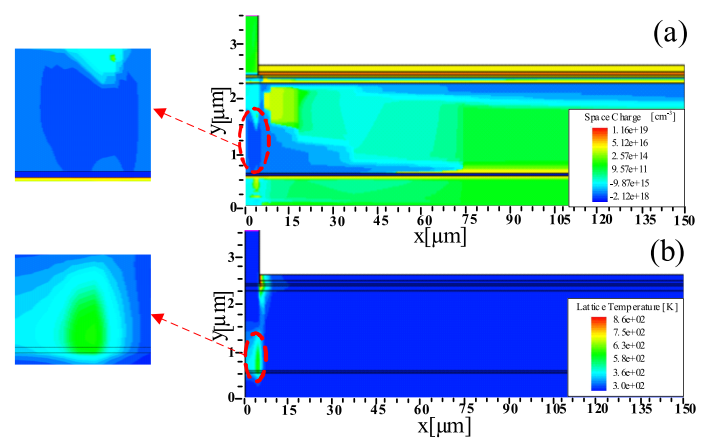

Fig. 5 The single-junction GaAs solar cell at 0.9 ns under the injection of HPMs signal with amplitude of $120 \mathrm{~V}$ and frequency of $2 \mathrm{GHz}$. (a) The space charge distribution inside the device. (b) The temperature distribution inside the device.

field, (located at the inflection point of the cathode) with its large curvature, high electric field, and carrier concentration higher than other regions, easily leads to a huge current density $\left(J_{n(p)}\right)$ and Joule heat accumulation (Fig. 4), which refers to Eqs. (1) and (2). However, due to the reverse bias of the largest PN junction, overall lower carrier concentration, and the absence of space charge region inside, it is difficult to realize avalanche breakdown at the front surface field.

In the negative half cycle (when the amplitude of the injected wave is less than zero), the largest PN junction between the emitter and the base is forward-biased, which leads to the current enhancement. Meanwhile, the depletion region of the back-surface field expands, resulting in the inversion of the base region, which then leads to an emergence of a large space charge region, due to the low doping concentration in the base region (Fig. 5). Finally, the high electric field and a huge current gather in the reverse-biased space charge region near the back-surface field, causing an increasing Joule heat and a high avalanche generation rate $(G)$, which refers to Eqs. (3) and (4).

\subsection{The burnout caused by Joule heat accumulation}

The burnout caused by Joule heat accumulation mainly occurs at the inflection point of the cathode front surface field (heterojunction), when a HPMs signal with a frequency above $3 \mathrm{GHz}$ is injected. The electric field intensity at the large curvature of the inflection point increases sharply, and the Joule heat that generated is much greater than the heat dissipation, causing the local temperature accumula-
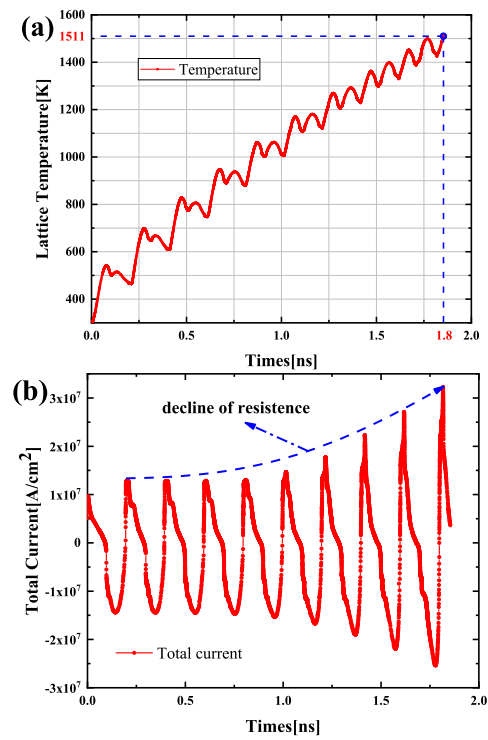

Fig. 6 Under the injection of HPMs signal with amplitude of $230 \mathrm{~V}$ and frequency of $5 \mathrm{GHz}$, the internal temperature and total current of the singlejunction GaAs solar cell. (a) Variation of peak temperature with time. (b) Variation of total current with time.

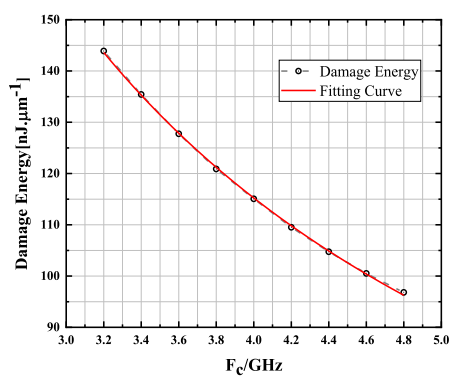

Fig. 7 Damage energy versus frequency, when the frequency of injection is above $3 \mathrm{GHz}$.

tion. Meanwhile, the material resistance decreases as temperature increases, further leading to an increase in current density $\left(J_{n(p)}\right)$ and intensification of Joule heat accumulation. Ultimately, the device burned out at $1.8 \mathrm{~ns}$ (Fig. 6).

Figure 7 illustrates the variation of damage energy with frequency under the HPMs injection with the same power but different frequency. The relationship between damage energy and the frequency of injection can be expressed by the following fitting formula:

$$
E=452.6 f_{c}^{-0.99}
$$

the correlation coefficient of Eq. (6) is 0.99, showing a high fitting degree. Furthermore, it reveals that the electric field becomes stronger and the hot carriers heat up more obviously, when injected with a higher frequency HPMs signal, leading to a faster burnout and less energy consumption.

\subsection{The burnout induced by avalanche breakdown}

As the HPMs signal with a frequency below $3 \mathrm{GHz}$ is injected, the high electric field and the hot carriers in the reverse-biased space charge region stay longer (due to the mobility degradation in high electric field), resulting in a high avalanche generation rate $(G)$. Finally, the avalanche multiplication effect occurs, and then leads to a sharp increase in the number of hot carriers, realizing the burnout 

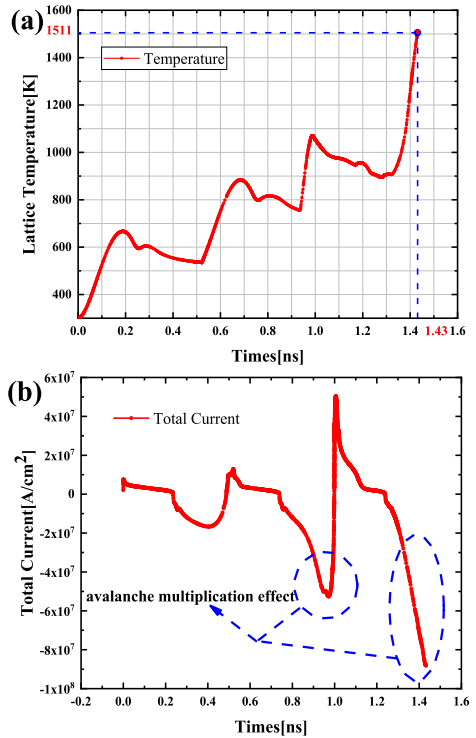

Fig. 8 Under the injection of HPMs signal with amplitude of $230 \mathrm{~V}$ and frequency of $2 \mathrm{GHz}$, the internal temperature and total current of the singlejunction GaAs solar cell. (a) Variation of peak temperature with time. (b) Variation of total current with time.

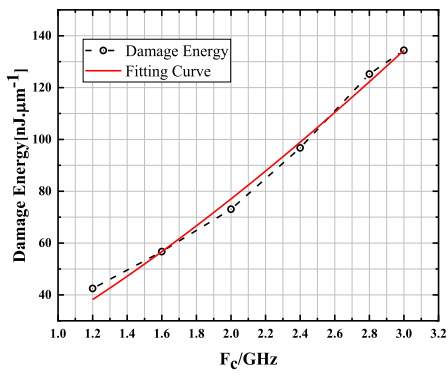

Fig. 9 Damage energy versus frequency, when the frequency of injection is below $3 \mathrm{GHz}$

of the device (Fig. 8).

The fitting variation of damage energy and the frequency of injection is depicted by the Fig. 9. The formula obtained by fitting is shown as follow:

$$
E=29.8 f_{c}^{1.37}
$$

the correlation coefficient of Eq. (7) is 1.37. Moreover, it demonstrates the difference between the two distinctive mechanisms of burnout. As the frequency of injection decreases, the drift time of the hot carrier in the reverse-biased space charge region near the back-surface field is prolonged, so the avalanche breakdown is more easily triggered.

\subsection{HPMs damage effects in the multi-junction GaAs solar cell}

Compared to the single-junction GaAs solar cell, the triplejunction GaAs solar shown in Fig. 1(b), is more difficult to burn out under the injection of the same HPMs. When a HPMs signal with amplitude of $120 \mathrm{~V}$ and frequency of $2 \mathrm{GHz}$ from the cathode port is injected, the number of space charge regions formed by the reverse bias of the backsurface field leaps; however, the space charge regions also have the effect of partial pressure, weakening the electric field strength and area of the reverse-biased space charge region [32]. Besides, due to the thickness of the battery
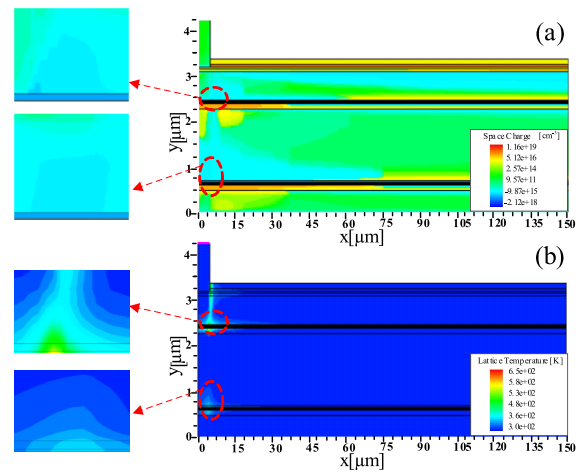

Fig. 10 The triple-junction GaAs solar cell at $0.9 \mathrm{~ns}$ under the injection of HPMs signal with amplitude of $120 \mathrm{~V}$ and frequency of $2 \mathrm{GHz}$. (a) The space charge distribution inside the device. (b) The temperature distribution inside the device.
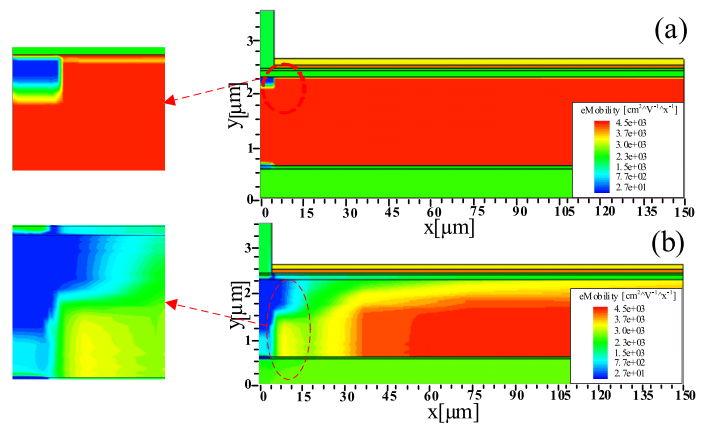

Fig. 11 The e-Mobility distribution in the single-junction GaAs solar cell. (a) The e-Mobility distribution in normal working state. (b) The e-Mobility distribution at $0.6 \mathrm{~ns}$ when injected HPMs with amplitude of $120 \mathrm{~V}$ and frequency of $2 \mathrm{GHz}$.

increases, heat dissipation is enhanced. Therefore, compared with the single-junction solar cell, as the same HPMs signal is injected into the multi-junction solar cell, the accumulation of Joule heat becomes slower, and the avalanche generation rate $(G)$ in the multi-junction GaAs solar cell also reduces (owing to the lesser accumulation of hot carriers and smaller area of reverse-biased space charge region), creating more difficulty in achieving burnout (Fig. 10 and Fig. 5).

\subsection{Soft damage effects on the performance of GaAs solar cell}

Analyzing the simulation results of the electron mobility inside the solar cell at multiple times and combining with Eq. (4), it can be concluded that when the injected HPMs signal does not reach the burnout threshold, the mobility $\left(\mu_{(E)}\right)$ decreases temporarily under the high electric field, causing recoverable damage (Fig. 11).

Solving the continuity equations and Possion's equation [33], the electron (hole) current expressions are expressed as follows:

$$
\begin{aligned}
& J_{n}=n q \mu_{n} E+q D_{n} \frac{\partial}{\partial x} n \\
& J_{\mathrm{p}}=p q \mu_{\mathrm{p}} E-q D_{\mathrm{p}} \frac{\partial}{\partial x} p
\end{aligned}
$$

in which $\mu_{n(p)}$ represents the mobility of electron (hole), and $D_{n(p)}$ indicates the diffusion coefficient of electron (hole). The following Eq. (10), known as the Einstein Relationship, 

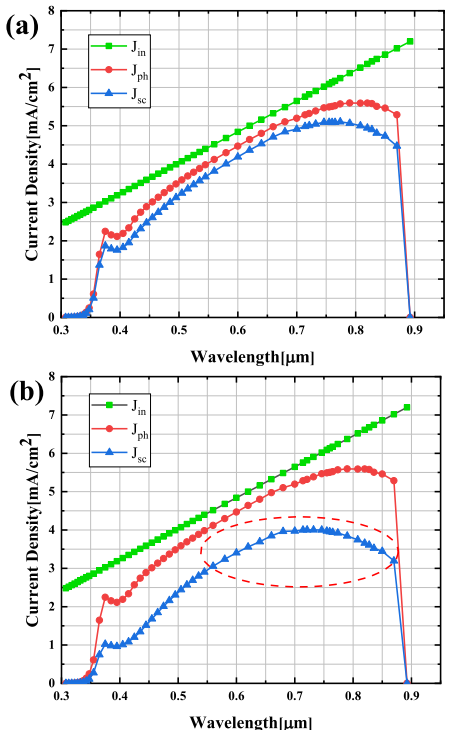

Fig. 12 Photon current density, photogenerated current density, and shortcircuit current density as a function of wavelength for the GaAs solar cell. (a) The normal station of GaAs solar cell. (b) The abnormal station of the equivalent model.

connects the mobility and the diffusion coefficient [34].

$$
D_{\mathrm{n}, \mathrm{p}}=\frac{k T}{q} \mu_{\mathrm{n}, \mathrm{p}}
$$

Combining Eqs. (8), (9) and (10), the relationship between the electron (hole) current and the mobility can be obtained as follows:

$$
\begin{aligned}
& J_{\mathrm{n}}=\left(p q E+k T \frac{\partial}{\partial x} n\right) \mu_{\mathrm{n}} \\
& J_{\mathrm{p}}=\left(p q E-k T \frac{\partial}{\partial x} p\right) \mu_{\mathrm{p}}
\end{aligned}
$$

The above Eqs. (11) and (12) demonstrate that the mobility degradation can cause a decrease in current. Therefore, based on the carrier mobility distribution in Fig. 11(b), an equivalent device model is rebuilt and reinjected into light with different wavelengths. Obviously, the short-circuit current $\left(J_{s c}\right.$, used to measure the ability to supply power to outside) drops apparently, which shows that the injection of HPMs signal affects the performance of the GaAs solar cell (Fig. 12).

\section{Conclusion}

This paper presents two-dimensional thermoelectric models of single-junction and triple-junction GaAs solar cells. By analyzing simulation results of the electric field, current density and temperature distribution of the devices, two different burnout mechanisms are proposed. In the positive half cycle, the high electric field at the inflection point of the cathode front surface field can trigger the burnout of GaAs solar cells, whereas during the negative half cycle, an avalanche multiplication effect could occur in the reversebiased space charge region near the back-surface field. Besides, by fitting the results under different conditions, two predictable formulas are obtained, which demonstrate that the two failure mechanisms are different in sensitivity to the frequency of injection. The damage caused by Joule heat accumulation occurs when the frequency is above $3 \mathrm{GHz}$, and damage energy decreases as the frequency increases; whereas the avalanche multiplication effect can be triggered when the frequency is below $3 \mathrm{GHz}$, and damage energy rises as the frequency rises. In addition, when compared to the single-junction solar cell, the multi-junction GaAs solar cell is more difficult to burn out under the same HPM interference, due to the enhancement of heat dissipation and the drop of avalanche ionization rate. Furthermore, an equivalent model (based on the carrier mobility distribution at the time of soft damage) is reinjected into the light with different wavelengths and compared with the light injection under normal conditions; it reveals that the mobility degradation caused by HPMs interference can affect the ability of solar cells to supply power to the outside.

\section{Acknowledgments}

Projected supported by the National Nature Science Foundation of China (No. 61974116).

\section{References}

[1] P.E. Glaser, et al:: "Power from the sun: its future," Science $\mathbf{1 6 2}$ (1968) 857 (DOI: 10.1126/science.162.3856.857).

[2] D. Cyranoski, et al.: "Japan sets sights on solar power from space," Nature 462 (2009) 398 (DOI: 10.1038/462398b).

[3] D. Cyranoski, et al.: "China sets sights on first solar power stations in space," Nature (2019) (DOI: 10.1038/d41586-019-00629-5).

[4] C. Dessert, et al.: "Interagency and international collaboration for space solar power implementation: a plan for space solar power project governance," IEEE International Conference on Wireless for Space and Extreme Environments (2013) (DOI: 10.1109/wisee. 2013.6737577).

[5] S. DebBarman, et al.: "A review: space based solar power (Sbsp) in development of smart city," SSRN Electronic Journal (2020) (DOI: 10.2139/ssrn.3621435).

[6] R.H. Nansen: "Wireless power transmission: the key to solar power satellites," IEEE Aerosp. Electron. Syst. Mag. 11 (1996) 33 (DOI: 10.1109/62.484148).

[7] W. Kaganov: "The transfer of energy from the solar-space power station to the earth," E3S Web of Conferences 25 (2017) 03003 (DOI: 10.1051/e3sconf/20172503003).

[8] J.N. Pelton, et al.: in Space 2.0 (Springer, 2019) 103-114 (DOI: 10.1007/978-3-030-15281-9_8).

[9] Y. Yang, et al.: "A novel design project for space solar power station (SSPS-OMEGA)," Acta Astronautica 121 (2016) 51 (DOI: 10.1016/ j.actaastro.2015.12.029).

[10] Z. Cheng, et al.: "In-orbit assembly mission for the space solar power station," Acta Astronautica 129 (2016) 299 (DOI: 10.1016/ j.actaastro.2016.08.019).

[11] G. Fan, et al.: "Thermal control strategy of OMEGA SSPS based simultaneous shape and topology optimization of butterfly wing radiator," International Communications in Heat and Mass Transfer 119 (2020) 104912 (DOI: 10.1016/j.icheatmasstransfer.2020.104912).

[12] National Research Council: Laying the Foundation for Space Solar Power: An Assessment of NASA's Space Solar Power Investment Strategy (National Academy Press, Washington D.C., 2001) (DOI: 10.17226/10202).

[13] D. Bienhoff: "Space infrastructure options for space based solar power," 6th International Energy Conversion Engineering Conference (IECEC) (2008) (DOI: 10.2514/6.2008-5618).

[14] J.L. Caton, et al.: "Space-based solar power: a technical, economic, and operational assessment," U.S. Army War College, Strategic Studies Institute (2015) (DOI: 10.21236/ada616385).

[15] J. Benford, et al:: High Power Microwaves (CRC Press, 2015) (DOI: 
10.1201/b19681).

[16] Y. Li, et al.: "Upset and damage mechanisms of the threedimensional silicon device induced by high power microwave interference," IEICE Electron. Express 16 (2019) 20190498 (DOI: 10.1587/ elex.16.20190498).

[17] H. Matsumoto: "Space solar power station (SSPS) and microwave power transmission (MPT)," 2003 IEEE Topical Conference on Wireless Communication Technology (2003) (DOI: 10.1109/ wct.2003.1321417).

[18] M. Furukawa, et al.: "5.8-GHz planar hybrid rectenna for wireless powered applications," 2006 Asia-Pacific Microwave Conference (2006) (DOI: 10.1109/apmc.2006.4429715).

[19] N. Shinohara and S. Kawasaki: "Recent wireless power transmission technologies in Japan for space solar power station/satellite," 2009 IEEE Radio and Wireless Symposium (2009) (DOI: 10.1109/ rws.2009.4957272).

[20] S. Sasaki and K. Tanaka: "Wireless power transmission technologies for solar power satellite," 2011 IEEE MTT-S International Microwave Workshop Series on Innovative Wireless Power Transmission: Technologies, Systems, and Applications (2011) (DOI: 10.1109/ imws.2011.5877137).

[21] C. Wang, et al.: "Design of rectifiers for high power wireless power transmission system," 2019 IEEE Wireless Power Transfer Conference (WPTC) (2019) (DOI: 10.1109/wptc45513.2019.9055624).

[22] A. Vijh, et al.: "High performance, lightweight GaAs solar cells for aerospace and mobile applications," IEEE 44th Photovoltaic Specialist Conference (PVSC) (2017) (DOI: 10.1109/pvsc.2017.8366342).

[23] X. Yu, et al:: "Analysis of high-power microwave induced degradation and damage effects in AlGaAs/InGaAs pHEMTs," Microelectronics Reliability 55 (2015) 1174 (DOI: 10.1016/j.microrel.2015.06.002).

[24] Y.-H. Zhang, et al.: "Modeling and understanding of the thermal failure induced by high power microwave in CMOS inverter," Chinese Physics B 26 (2017) 058502 (DOI: 10.1088/1674-1056/26/5/058502).

[25] H. Li, et al.: "Damage effects and mechanism of the silicon NPN monolithic composite transistor induced by high-power microwaves," Chinese Physics B 27 (2018) 088502 (DOI: 10.1088/ $1674-1056 / 27 / 8 / 088502)$.

[26] R. van Overstraeten and H.De Man: "Measurement of ionization rates in diffused silicon $p$ - $n$ junctions," Solid-State Electronics 13 (1970) 583 (DOI: 10.1016/0038-1101(70)90139-5).

[27] N.D. Arora, et al.: "Electron and hole mobilities in silicon as a function of concentration and temperature," IEEE Trans. Electron Devices 29 (1982) 292 (DOI: 10.1109/t-ed.1982.20698).

[28] C.R. Valenta and G.D. Durgin: "Harvesting wireless power: survey of energy-harvester conversion efficiency in far-field, wireless power transfer systems," IEEE Microw. Mag. 15 (2014) 108 (DOI: 10.1109/MMM.2014.2309499).

[29] E.C.T. Kho, et al.: "200-V lateral superjunction LIGBT on partial SOI," IEEE Electron Device Lett. 33 (2012) 1291 (DOI: 10.1109/ LED.2012.2205892).

[30] M. Nand, et al.: "Development of high damage threshold multilayer thin film beam combiner for laser application," Dae Solid State Physics Symposium 2015 (2016) (DOI: 10.1063/1.4947929).

[31] M. Masuda, et al.: "Reduce of electric near-field intensity by a shield box in wireless power transfer via field resistance coupling," 2015 IEEE International Telecommunications Energy Conference (INTELEC) (2016) 2158 (DOI: 10.1109/INTLEC.2015.7572436).

[32] S.M. Sze and K.K. Ng: Physics of Semiconductor Devices (John Wiley \& Sons, 2006) (DOI: 10.1002/0470068329).

[33] P. Würfel: Physics of Solar Cells: from Principles to New Concepts (John Wiley \& Sons, 2005) (DOI: 10.1002/9783527618545).

[34] M.P. Allen and A.J. Masters: "Some notes on Einstein relationships," Molecular Physics 79 (1993) 435 (DOI: 10.1080/ 00268979300101331). 